\section{Sun protection factor of South African-tested sunscreens}

To the Editor: This letter seeks to to shed some light and rationality on the recent confusion surrounding the Cancer Association of South Africa (CANSA)-initiated ultraviolet A (UVA) test results on some South African (SA) sunscreens.

In August 2012, media publicity questioned the efficacy and safety of SA-produced sunscreens, suggesting that locally produced sunscreen products 'didn't meet standards'. This controversy originated from a small-scale in vitro UVA protection study that was conducted for CANSA on just $10 \%$ of products sold in SA.

The in vivo sun protection factor (SPF) of the products was never in question, despite suggestions to the contrary in the popular media (and even in SAMF). The SA SPF test (SANS 1557), which is conducted on healthy human volunteers, is reliable and has been in line with the International Standards Organisation (ISO) SPF in vivo test (ISO 24444) throughout the latter's development. In fact SA played a key role in initiating the ISO standard in 2006.

Take-home point 1: The SPF of tested SA sunscreens is reliable so use them.

The current controversy surrounds the UVA/ultraviolet B (UVB) balance of protection of the sunscreens. In late May the ISO 24443 in vitro UVA test was published and is due to be incorporated into the SANS, but is not yet in any SA National Standard. The ISO 24443 is an instrumental test - not an in vivo test - that seeks to test for UV filter photodegradation. Products are irradiated with a full UVA and UVB spectrum equivalent to the in vivo SPF of the sunscreen (e.g. SPF 30 or 50) to 'stress' the sunscreen product and test for potential photodegradation. Thereafter, the UVA/UVB protection spectrum is tested. The new ISO standard (24443) for in vitro UVA tests requires the post-irradiation UVB/UVA in vitro ratio to be greater than 1:0.3.

The SANS 1557 states only that the UVB/UVA protection ratio must exceed 1:0.4. There is currently no SANS for in vitro UVA, although a process was included in earlier editions of SANS 1557.

All the CANSA-tested products complied with the existing SPF, UVA and irritancy requirements at the time of going to market. The test that was conducted (and incorrectly reported in the media as the 'COLIPA' test) produced post-irradiation results for some of the tested products below the ISO-stated 1:0.3 UVB/UVA ratio. For an SPF 30 product, the UVA protection factor (UVAPF) was therefore less than 9, or for an SPF 50, less than 15.

Take-home point 2: All products tested had SPF and UVA test data that complied with existing standards.

The most effective and widely used UVA filter (butylmethoxydibenzoyl methane) is prone to some photodegradation, as is also the case for one of the most commonly used UVB filters, (octyl methoxycinnamate), albeit to a lesser extent. These two filters can be photostabilised with the addition of any of three other ultraviolet (UV) filters (octocrylene, methylbenzylidine camphor or diethylhexyl naphthalate). The organic pigments, such as zinc oxide and titanium dioxide, are not known to photodegrade.

Take-home point 3: Products can be stabilised against photodegradation, and photodegradation does not occur with the inorganic sunscreen pigments.

Dermatologists agree that the link between UVA exposure and melanoma is tenuous. Melanoma is genetically linked, and while UV exposure certainly plays a role in its causation, evidence is conflicting in respect of the significance of UVA exposure. Moreover, the daily UVAPF protection factor requirement is unknown. However, UVA levels in SA are not thought to typically exceed 7 minimal erythemal doses (MEDs) a day.

Take-home point 4: The UVA protection factor required to protect against skin damage is not known. While UVA levels in SA are not thought to exceed 7 UVAPFs a day, an SPF 30 product would have to have UVAPF of $>7.5$ to pass the ISO test.

High SPFs cannot be achieved without some UVA protection, as the SPF in vivo test is inherently a UVA and UVB protection test. The products tested did provide protection against both UVA and UVB. The maximum UV exposure possible, dawn to dusk, on a clear sunny summer's day in SA is 35 MEDs. It is surely unlikely that any sunconscious individual would stay out the whole day and fail to reapply sunscreen regularly as recommended!

Take-home point 5: The in vivo sun protection factor (SPF) of the sample of products tested on healthy human volunteers is reliable, but they must be re-applied regularly as recommended ... sunscreens are designed to be used regularly and often.

The CANSA seal of recognition remains a trustworthy guide to quality and reliability. It is safer to use sunscreens than not to do so!

Beverley Summers

Photobiology Laboratory, Department of Pharmacy, University of Limpopo (Medunsa Campus), North-West

S Afr Med J 2012;102(12):897. DOI:10.7196/SAMJ.6428 